\title{
Challenges With Implementation of a Respiratory Therapist-Driven Protocol of Spontaneous Breathing Trials in the Pediatric ICU
}

\author{
Conrad Krawiec MD, Dale Carl RRT, Christy Stetter, Lan Kong PhD, Gary D Ceneviva MD, and \\ Neal J Thomas MD
}

\begin{abstract}
BACKGROUND: Timely ventilator liberation is crucial in the pediatric ICU. In many pediatric ICUs, the decision to initiate weaning is driven by the physician, which may lead to delays in ventilator liberation. The objectives of this quality improvement project were to develop and implement a respiratory therapist (RT)-led protocol for screening for spontaneous breathing trial (SBT) readiness, to test protocol feasibility, and to evaluate its impact on SBT timing. METHODS: A retrospective chart review was performed on all intubated patients in the pediatric ICU for 18 months prior to protocol institution. An RT-driven protocol was developed and implemented, enabling consistent screening for SBT readiness. When criteria were met, an SBT was initiated after order placement. The difference in the timing of the first SBT between physician-directed screening and the RT-driven protocol was evaluated. RESULTS: A total of 219 subjects were included in this project (128 pre-intervention; 91 intervention). Baseline demographic data, including mortality risk and endotracheal tube size, were similar in both groups. The time of the first SBT (median [25th, 75th percentile]) was not significantly different between the intervention and preintervention groups $(39.5$ [25.3, 85.2] vs 42.6 [26.4, 81.3], respectively). There was no difference in mechanical ventilation duration, or length of hospital and ICU stay. The odds of being placed on noninvasive respiratory support were higher in the intervention group at $1 \mathrm{~h}$ (odds ratio [95\% CI]: 2.29 [1.10, 4.78], $P=.03$ ) and $12 \mathrm{~h}$ (odds ratio $2.53[1.23,5.20], P=.01)$ postextubation, but the odds of re-intubation did not reach statistical significance (odds ratio $2.60[0.73,9.27], P=.14$ ). RT adherence with patient screening was $56.4 \%$. CONCLUSIONS: An RT-driven protocol was successfully introduced in an academic pediatric ICU. However, it did not impact time of SBT initiation, potentially due to the difficulty in maintaining adherence over time. RT-driven protocols require further study. Key words: ventilator weaning; respiratory therapy; quality improvement; clinical protocol; physician's role; pediatrics. [Respir Care 2017;62(10):1233-1240. (C) 2017 Daedalus Enterprises]
\end{abstract}

Introduction

Pediatric critical care requires the simultaneous management of multi-organ system dysfunction, including con-

\footnotetext{
Dr Krawiec and Dr Ceneviva are affiliated with the Pediatric Critical Care Medicine, Department of Pediatrics, Penn State Milton S. Hershey Medical Center, Penn State Hershey College of Medicine, Penn State Hershey Children's Hospital, Hershey, PA. Mr. Carl is affiliated with Penn State Health Respiratory Care and Pulmonary Diagnostics, Penn State Health Children's Hospital, Hershey, PA. Dr Kong and Ms Stetter, as well as Dr Thomas, are affiliated with the Department of Public Health Sciences, Pennsylvania State University College of Medicine, Hershey, Pennsylvania.
}

stant assessment and alterations in mechanical ventilation. Once illness recovery commences, liberation from the ventilator should be considered as soon as possible because

\footnotetext{
The project was supported by the National Center for Advancing Translational Sciences, National Institutes of Health, through Grant UL1 TR000127 and TR002014. The content is solely the responsibility of the authors and does not necessarily represent the official views of the NIH.

Correspondence: Conrad Krawiec MD, Department of Pediatrics, Penn State Hershey Children's Hospital, 500 University Drive, PO Box 850, Hershey, PA 17033-0850. E-mail: ckrawiec@ hmc.psu.edu.
}

DOI: $10.4187 /$ respcare. 05477 
prolonged mechanical ventilation is associated with an increased risk of ventilator-associated complications. ${ }^{1-5}$

See the Related Editorial on Page 1368

Previous studies in adults have demonstrated that a delay in the assessment of a patient's readiness to wean or initiation of a spontaneous breathing trial (SBT) can contribute to prolonged mechanical ventilation. ${ }^{6,7}$ Because critically ill pediatric patients are becoming increasingly complex, continuous assessment of a patient's ability to spontaneously breathe is paramount to the care of patients who are recovering from their critical illness. ${ }^{8}$ This is especially important because it was recently suggested that pediatric patients who pass a daily extubation readiness screening have a higher likelihood of successful extubation. ${ }^{9}$

Most studies on pediatric ventilator weaning compare protocol versus usual care with the physician primarily performing the screening. ${ }^{1,10-12}$ While the largest study to date showed a significant decrease in duration of mechanical ventilation, the study also implies that pediatric critical care physicians continue to take an active role in assessing recovering patients and determining readiness to wean. ${ }^{10}$ Because a limited number of providers are required to assess and manage many patients, delays in assessment are likely.

In the literature and practice related to critically ill adults, respiratory therapists (RTs) have been utilized to identify patients who are capable of breathing spontaneously; studies have demonstrated improved patient outcomes, including a decrease in duration of mechanical ventilation, with the use of protocols. ${ }^{6,7,12}$ A review of the literature reveals only one study that compared the use of RT-driven protocols to facilitate pediatric mechanical ventilator weaning versus usual care. ${ }^{12}$ The impact of an RT-driven protocol in the pediatric ICU (PICU) is unknown.

In the PICU at Penn State Children's Hospital, it was observed that physicians decide when to initiate SBTs during rounds. Depending on the acuity of the unit and when an intubated patient is rounded on, SBTs potentially can be delayed. There is an interest to develop methods to decrease mechanical ventilation duration, but the rigidity of a randomized controlled trial to allow RTs to perform a task often performed by the pediatric critical care physician could cause a disturbance in provider workflow. Therefore, we opted to perform a quality improvement project to allow us to study the process of implementing a protocol and make adjustments as necessary.

The primary aims of this quality improvement project were to standardize a method of early non-physician recognition of SBT readiness, to establish the feasibility of using an RT-driven protocol for these SBTs, and to determine if SBTs occur earlier with an RT-driven protocol in a single high-acuity academic PICU.

\section{QUICK LOOK}

\section{Current knowledge}

In the literature pertaining to critically ill adults, a delay in the assessment of a patient's readiness to wean or initiate spontaneous breathing trials can contribute to prolonged mechanical ventilation. There is very little evidence supporting the true impact of a respiratory therapist-driven protocol in the pediatric ICU.

\section{What this paper contributes to our knowledge}

A respiratory therapist-driven protocol was implemented in an academic pediatric ICU where medical decisions are primarily driven by physicians. Protocol adherence was difficult to maintain over time, and it did not impact time to spontaneous breathing trial initiation.

\section{Methods}

\section{Quality Improvement Project Design}

This was a pre- and postintervention quality improvement project focused on mechanically ventilated pediatric patients admitted to the PICU at Penn State Hershey Children's Hospital. A retrospective review was completed in the 18 months prior to implementation of an RT-based SBT protocol, and the findings of the retrospective review were compared with data from 12 months after implementation. The study was approved by the institutional review board. This study did not meet the definition of human research according to the IRB as the data were not obtained through interaction or an intervention with the individual, and we did not obtain or use identifiable private information.

\section{Usual Care Definition/Mechanical Ventilation and Weaning Practices}

Our unit is usually staffed with 2 RTs and can be flexed up to 3 RTs depending on patient census. A core group of 30 RTs routinely practice in the PICU. Usual care practice is that all ventilated patients admitted to the PICU are placed on the synchronized intermittent mandatory ventilation-pressure-regulated volume control (SIMV-PRVC) mode of mechanical ventilation, with a pressure support set at the discretion of the RT. Most commonly, regardless of endotracheal tube (ETT) size, the pressure support is set at $5 \mathrm{~cm} \mathrm{H}_{2} \mathrm{O}$ unless the patient clinically requires a higher level. Ventilator settings are determined at the preference of the attending physician with constant communication with the respiratory therapy team. 
The decision to initiate SBTs is commonly made during physician rounds, which occur from 8:00 AM until noon each day. Prior to implementation, no formal screening process existed, and any decision to initiate an SBT was made at the discretion of the attending physician; at times, PICU fellows or nurse practitioners would initiate an SBT if this was not addressed by the rotating pediatric resident. Once the decision to initiate an SBT is made, an order for the trial is placed to electronically notify the RT. The RT evaluates the patient, confirms appropriateness, and ensures the presence of a bedside nurse. The initial pressure support is generally set at $5 \mathrm{~cm} \mathrm{H}_{2} \mathrm{O}$ with a PEEP of 5, and the trial routinely lasts for $2 \mathrm{~h}$. Repeat trials and the decision to extubate the patient is made at the discretion of the critical care team under direct supervision of the attending physician. An order for the level of sedation as outlined by the Penn State Children's Hospital Sedation Algorithm is required for all mechanically ventilated patients. ${ }^{13}$

\section{Protocol Development}

This protocol was developed in conjunction with our Penn State Hershey Children's Hospital respiratory care clinical specialist with input from the pediatric critical care team, which includes physicians, nurse practitioners, and fellows. This process consisted of multiple drafts and revisions and incorporated various clinical parameters and screening criteria based on an extensive literature review of adult and pediatric ventilator weaning strategies. ${ }^{6,7,9-11,14,15}$ We ensured that the RT would have the ability to objectively measure a patient's clinical stability, oxygenation, ventilation, and mental status prior to initiation of an SBT. The pediatric sedation score of the patient was taken into account; if the score was noted to be $>3$, the patient was not consistently breathing above the ventilator and thus was deemed not appropriate for SBT. ${ }^{13}$

The ventilator mode for the SBT was chosen on the basis of usual clinical practice. The pressure support settings were adjusted for ETT size and the associated increase in resistance with a smaller diameter tube. ${ }^{11,16}$ While controversial, when compared to previous studies performed on this topic, adjustment of pressure support by ETT size was the most utilized. ${ }^{9-11}$

\section{Protocol Implementation}

Once the clinical parameters and algorithm were agreed upon, an SBT worksheet was developed (see online supplemental material at www.rcjournal.com). As the pediatric respiratory therapy team was familiar with an algorithm already in place at the Penn State Hershey Medical Center adult medical and surgical intensive care units, the goals and a draft of the worksheet were communicated prior to protocol implementation. Furthermore, during the month prior to protocol initiation, the worksheet was informally discussed multiple times with respiratory therapy team members. Physician and nursing leaders of the PICU were given a copy of the protocol, and it was discussed during their respective staff meetings prior to protocol implementation.

The protocol was initiated on June 1, 2014, and included all patients (with the exception of pediatric cardiothoracic surgery service) who were admitted to the PICU and were mechanically ventilated. The critical care team managed all patients and supervised the respiratory therapy management of the protocol. The worksheet was printed and was readily available for RTs in the unit. Screening times occurred at 7:30 AM and 7:30 PM. The times were chosen for patient safety to ensure a provider skilled in pediatric airway management was available as sedation is usually decreased during an SBT to facilitate spontaneous breathing, thus increasing the risk of self-extubation. A 12-h interval was chosen to limit the burden of the protocol and facilitate adherence as our RTs perform other tasks. Initiation of an SBT was not considered until after the RT reviewed the worksheet and ensured appropriateness. Furthermore, placement of an electronic order for an SBT was required after a discussion between the RT and the critical care team. During the first 2 weeks of protocol implementation, RTs initiated SBTs without discussion or orders from the critical care team if the agreed-upon criteria were met, but this was altered due to the constraint of the RT not being able to place a verbal order and to electronically document SBT appropriateness.

\section{Subject Population}

The PICU at Penn State Hershey Children's Hospital is an 18-bed, tertiary care facility in which medical, general surgical, and cardiothoracic patients are treated. All patients who were mechanically ventilated $>24 \mathrm{~h}$ at Penn State Children's Hospital PICU and were $\leq 18$ y old were included in the quality improvement project. This population consisted of subjects who were intubated in-house or from an outside facility for acute respiratory failure as well as subjects who were kept intubated or were re-intubated after a surgical procedure. The start of mechanical ventilation time was determined by reviewing the electronic medical record and recording RT documentation of ventilation initiation in the PICU for all patients. For subjects who were intubated in the operating room, the start of mechanical ventilation time was when the subject was documented as being intubated by the anesthesiologist. Patients were excluded if they (1) were admitted to pediatric cardiothoracic surgery, (2) were intubated secondary to airway issues, (3) were undergoing brain death determination, (4) had do-not-resuscitate orders, (5) were mechanically ventilated via tracheostomy, or (5) had an SBT 
before being screened by respiratory therapy. In addition, if a subject was re-intubated within $72 \mathrm{~h}$ after extubation, the second occurrence of mechanical ventilation was not included.

\section{Data Collection}

In the pre-intervention phase, a census was obtained for all patients admitted to the PICU from February 1, 2013, until May 31, 2014. We reviewed the medical record and determined eligibility based on the above inclusion and exclusion criteria. For the intervention phase, the medical records of all patients admitted in the PICU between June 1, 2014, and May 31, 2015, were reviewed to determine the number of mechanically ventilated patients who qualified for screening. The adherence percentage was calculated by comparing the number of patients who were mechanically ventilated to the number of patients actually screened by respiratory therapy. Of the patients who were screened by respiratory therapy, we reviewed the medical record to ascertain which patients qualified for screening on the basis of the above inclusion and exclusion criteria. There was no run-in period before the quality improvement project to evaluate for any issues with the protocol.

To fully assess respiratory therapy protocol adherence patients who were not eligible for screening but who were still screened and underwent an SBT were included. However, these subjects were excluded from the final analysis due to either being intubated for $<24 \mathrm{~h}$ or due to a predefined protocol violation (eg, PEEP $>5$, sedation level $>3$, etc.). Data collected included demographic (age, sex, height, weight, body surface area, ETT size, admission diagnoses) information as well as Pediatric Risk of Mortality III and Pediatric Index of Mortality 2 scores from the Virtual PICU Systems database (Virtual PICU Systems, Los Angeles, CA). We analyzed outcome, process, and balancing measures. The outcome measures that we analyzed included time from initiation of invasive mechanical ventilation to SBT, time from initiation of SBT to extubation, duration of invasive mechanical ventilation, length of hospital stay, and length of ICU stay. Process measures that were tracked included the number of paper sheets that were filled out by the respiratory therapy team compared to eligible patients on invasive ventilator support. Balancing measures included the use of noninvasive respiratory support (eg, bi-level positive airway pressure, CPAP, and high-flow nasal cannula) after extubation and re-intubation.

\section{Statistical Analysis}

Demographic data were compared between the preintervention and intervention groups using a Wilcoxon test for continuous data and a chi-square test for categor- ical data. An analysis of covariance model was used to compare the pre-intervention and intervention groups, adjusting for Pediatric Risk of Mortality III, Pediatric Index of Mortality 2, and ETT size. Distributions of outcomes were examined and appropriate transformations were applied as needed prior to running the analysis of covariance model. For ease of interpretation, results are reported as median (25th percentile, 75th percentile) for each group, while the reported $P$ value is from the analysis of covariance model. For the binary outcomes (ie, oxygen therapy, re-intubation), a logistic regression model adjusting for Pediatric Risk of Mortality III, Pediatric Index of Mortality 2, and ETT size was used to compare the 2 groups, with the effect size reported as an odds ratio $(95 \% \mathrm{CI})$. All tests were performed using SAS version 9.4 (SAS Institute, Cary, NC) at a significance level of 0.05 .

\section{Results}

\section{Subject Demographics}

A total of 725 medical records were reviewed and 128 subjects were included in the pre-intervention arm of the quality improvement project. For the intervention phase, a total of 650 patients were reviewed, of whom 249 mechanically ventilated patients qualified for screening. Of these, 133 subjects $(53.4 \%)$ were screened by respiratory therapy. Forty-two patients who were not eligible for screening, but were screened by respiratory therapy and underwent an SBT, were included to fully assess respiratory therapy adherence. Ninety-one subjects met eligibility criteria and were included in the intervention arm. Baseline demographic data were similar in both groups including risk of mortality and ETT size (Table 1). A respiratory diagnosis comprised $49 \%$ of the pre-intervention group and $35 \%$ of the intervention group.

\section{Initiation of an SBT and Extubation Practices}

The time from initiation of mechanical ventilation to the first SBT (shown as median [25th percentile, 75th percentile]) was similar between the intervention group and the pre-intervention group (39.5 h [25.3, 85.2] vs $42.6 \mathrm{~h}$ [26.4, 81.3], $P=.53)$, as was time to extubation after initiation of the SBT $(49.5 \mathrm{~h}[14.5,74.3]$ vs $41.3 \mathrm{~h}$ [19.6, 71.1], $P=.25$ ) (Table 2). Not unexpectedly, a higher Pediatric Risk of Mortality III score was associated with a longer time from initiation of mechanical ventilation to SBT $(P<.001)$ and a longer time to extubation after SBT initiation $(P<.001)$. The presence of a larger ETT size had no effect on time to initiation of SBTs $(P=.16)$, but was associated with shorter time to extubation after initiation of SBTs $(P=.001)$. 


\section{Duration of Mechanical Ventilation and Length of Hospital and ICU Stay}

Duration of mechanical ventilation $(100.0 \mathrm{~h}[49.5,170.5]$ vs $92.8 \mathrm{~h}[46,154.7], P=.69)$ and length of PICU stay (6.4 d [3.6, 13.0] vs $6.4 \mathrm{~d}[3.7,10.7], P=.44)$ were similar between the 2 groups. There was a trend toward longer hospital stay in the intervention group (12.5 d [9.0,

Table 1. Demographics

\begin{tabular}{lccr}
\hline \hline & $\begin{array}{c}\text { Pre-intervention } \\
(n=128)\end{array}$ & $\begin{array}{c}\text { Intervention } \\
(n=91)\end{array}$ & $P$ \\
\hline Age, months* & $33.7(3.5,157.7)$ & $24.2(3.4,137.7)$ & .77 \\
Sex, \% male (male:female) & $56 \%(72: 56)$ & $49 \%(45: 46)$ & .32 \\
Height, cm* & $92.0(54.0,132.1)$ & $84.0(58.0,136.0)$ & .88 \\
Weight, kg* & $13.8(4.7,35.8)$ & $11.9(5.3,30.0)$ & .72 \\
Body surface area, ${ }^{2 *}$ & $0.6(0.3,1.2)$ & $0.5(0.3,1.1)$ & .88 \\
Endotracheal tube size, cm* & $4.5(3.5,6.0)$ & $4.0(3.5,6.0)$ & .48 \\
PIM* & $-3.4(-4.6,-3.0)$ & $-3.4(-4.4,-3.0)$ & .82 \\
PRISM* & $7.0(3.0,12.0)$ & $7.0(3.0,10.0)$ & .62 \\
Admission diagnosis, $n(\%)$ & & & .20 \\
Cardiac & $14(10.9)$ & $8(8.8)$ & \\
Neurologic & $20(15.6)$ & $18(19.8)$ & \\
Respiratory & $63(49.2)$ & $32(35.2)$ & \\
Sepsis & $4(3.1)$ & $2(2.2)$ & \\
Surgery & $13(10.2)$ & $12(13.2)$ & \\
Trauma & $14(10.9)$ & $19(20.9)$ & \\
& &
\end{tabular}

* Data reported as median (25th, 75 th percentile).

PIM $=$ Pediatric Index of Mortality 2

PRISM $=$ Pediatric Risk of Mortality III
21.6] vs $12.1 \mathrm{~d}[7.9,18.6], P=.08)$, but this did not reach statistical significance. These results are summarized in Table 2.

\section{Adverse Events}

The odds of being placed on any form of noninvasive respiratory support at $1 \mathrm{~h}$ postextubation were higher in the intervention group (odds ratio [95\% CI]: 2.29 [1.10, 4.78], $P=.03)$. At $12 \mathrm{~h}$ postextubation, this effect was still seen (odds ratio [95\% CI]: $2.53[1.23,5.20], P=.01$ ). The odds of being re-intubated after extubation was not statistically different between the 2 groups (odds ratio [95\% CI]: 2.60 [0.73, 9.27], $P=.14$ ) (Table 3).

\section{Respiratory Therapy Protocol Adherence}

Table 4 summarizes adherence to the protocol. Respiratory therapy screened $56.4 \%$ of patients eligible for screening. They adhered to established screening times $30.7 \%$ of the time. They were compliant with the established initial pressure support level noted in the algorithm $42.1 \%$ of the time. Figure 1 shows a very high adherence rate in the beginning of protocol implementation, with a noted drop off in December 2014 and January 2015.

\section{Discussion}

We hypothesized that, with protocol-directed guidance and extending more autonomy, RTs would screen a majority of

Table 2. Outcome Data

\begin{tabular}{|c|c|c|c|}
\hline & Pre-intervention* $(n=128)$ & Intervention* $(n=91)$ & $P^{* *}$ \\
\hline Duration of mechanical ventilation, $\mathrm{h}$ & $92.8(46,154.7)$ & $100.0(49.5,170.5)$ & .69 \\
\hline Initiation of ventilation to initiation of SBT, $h$ & $42.6(26.4,81.3)$ & $39.5(25.3,85.2)$ & .53 \\
\hline Initiation of SBT to extubation, $\mathrm{h}$ & $41.3(19.6,71.1)$ & $49.5(14.5,74.3)$ & .25 \\
\hline Hospital length of stay, $d$ & $12.1(7.9,18.6)$ & $12.5(9.0,21.6)$ & .08 \\
\hline ICU length of stay, $d$ & $6.4(3.7,10.7)$ & $6.4(3.6,13.0)$ & .44 \\
\hline
\end{tabular}

* Data reported as median (25th, 75th percentile).

** $P$ value from an analysis of covariance model adjusting for Pediatric Risk of Mortality III, Pediatric Index of Mortality 2, and endotracheal tube size. Square root transformation was applied to initiation of SBT to extubation, and log transformation was applied to all other outcomes.

$\mathrm{SBT}=$ spontaneous breathing trial

Table 3. Oxygen Therapy and Re-Intubation Rates

\begin{tabular}{|c|c|c|c|c|}
\hline & \multirow{2}{*}{ Pre-intervention, $n(\%)$} & \multirow{2}{*}{ Intervention, $n(\%)$} & \multicolumn{2}{|c|}{$\begin{array}{l}\text { Intervention vs. } \\
\text { Pre-intervention* }\end{array}$} \\
\hline & & & Odds Ratio $(95 \% \mathrm{CI})$ & $P$ \\
\hline Noninvasive respiratory support ( $1 \mathrm{~h}$ after extubation) & $15(6.9 \%)$ & $21(9.6 \%)$ & $2.29(1.10,4.78)$ & .03 \\
\hline Noninvasive respiratory support ( $12 \mathrm{~h}$ after extubation) & $18(8.7 \%)$ & $23(11.1 \%)$ & $2.53(1.23,5.20)$ & .01 \\
\hline Re-intubation & $4(1.8 \%)$ & $7(3.2 \%)$ & $2.60(0.73,9.27)$ & .14 \\
\hline
\end{tabular}


Table 4. Respiratory Therapist-Driven Protocol Adherence

\begin{tabular}{lc}
\hline \hline Intubated patients eligible for screening, $n$ & 133 \\
Intubated subjects screened by respiratory & $75 / 133(56.4 \%)$ \\
$\quad$ therapy, $n / N(\%)$ & \\
Respiratory therapy adhered to established & $23 / 75(30.7 \%)$ \\
$\quad$ screening times, no./total (\%) & $56(42.1 \%)$ \\
Respiratory therapy adherent with established & \\
$\quad$ initial pressure support level, no./total (\%) & \\
\hline
\end{tabular}

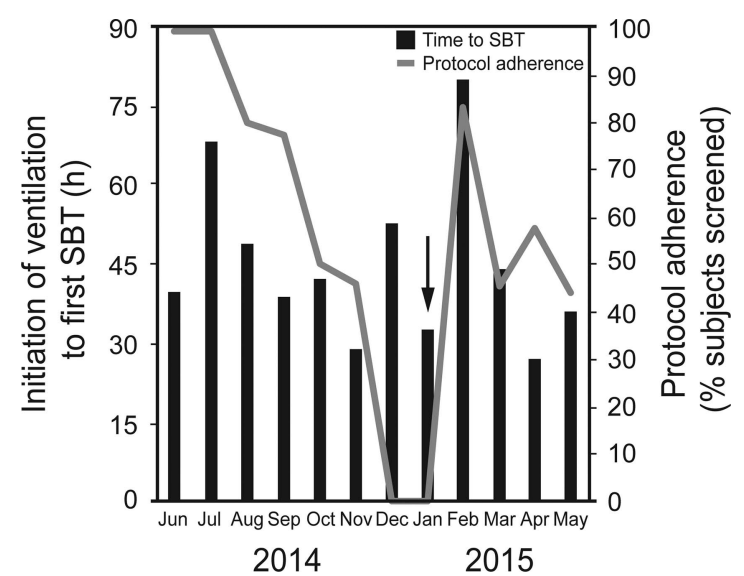

Fig. 1. Time from initiation of mechanical ventilation to first SBT and Respiratory therapist-driven protocol adherence (\% subjects screened). Arrow indicates data sharing and communication with senior respiratory therapy leaders. Respiratory therapy leaders then immediately discussed and reinforced the importance of protocol adherence during a respiratory care department staff meeting. $\mathrm{SBT}=$ spontaneous breathing trial.

these patients, determine SBT eligibility, and impact the timing of the first SBT. This quality improvement project introduced an RT-driven SBT protocol in an academic center, but this protocol did not impact the time of SBT initiation, possibly due to decreased protocol adherence.

To date, the only randomized, controlled trial to evaluate an RT-driven weaning protocol was performed by Schulz et al. ${ }^{12}$ That study, which utilized an algorithmbased weaning strategy with active titration of the pressure support level by RTs, demonstrated a reduction in weaning time. Subsequent studies utilized different screening criteria, extubation readiness testing, and clinician screeners. Randolph et al, ${ }^{11}$ for example, used a minimal pressure support based on ETT size as a screening strategy to randomize subjects to one of 3 different weaning protocol groups (ie, pressure support, volume support, no protocol). Foronda et al, ${ }^{10}$ performing the largest study to date, utilized physicians who screened and initiated a 2-h SBT to assess extubation readiness. These studies used similar screening criteria, but a recent secondary analysis of data from the Randomized Evaluation of Sedation Titration for Respiratory Failure clinical trial used a simplified nursingdriven protocol to screen for spontaneous breathing and an oxygenation index or oxygenation saturation index of $\leq 6 .{ }^{9}$ While these studies are useful and do suggest that protocols using extubation readiness testing or weaning reduce the duration of mechanical ventilation, ${ }^{9,10}$ there is a lack of consensus on the best approach. More studies evaluating RT-driven protocols to provide early identification of pediatric patients eligible for ventilator liberation are necessary.

When compared to previous pediatric studies, this quality improvement project had a unique protocol and understanding of the culture change that can occur with this change in clinical practice. Our project used an RT-driven protocol utilizing a 2-h SBT with specific minimal pressure support settings, and any patient who passed was referred to the critical care team to facilitate earlier physician recognition of extubation readiness. This project was intentionally not performed as a randomized controlled trial to allow all critical care team members to become accustomed to their new roles. To our knowledge, this is the first time such an approach has been described.

The protocol was successfully introduced, as shown by the high initial RT adherence rates, suggesting interest in this new clinical practice. Maintaining this level of adherence was difficult, however, due to several barriers encountered during the course of this project. This quality improvement project tested a protocol designed to provide objective criteria to a practice that is historically subjective. Because the final clinical decision fell on the critical care team, the objectivity of the assessment may have been weakened, making communication and approachability difficult. PICUs rely heavily on the RT to ensure pulmonaryrelated tasks are completed, including suctioning, mechanical ventilator setup and monitoring, and administration of aerosol medications. If patient acuity increases, such as during months of high respiratory virus incidence, the protocol may have become a lower priority as RTs often must triage tasks in order of importance. We did note, however, an increase in the adherence rate after we shared the midproject adherence results with senior leaders of the respiratory care department; these leaders reminded clinical staff during a staff meeting of the importance of this protocol as well as the understanding that all efforts should be made to follow this protocol. This highlights the value of constantly observing and studying the results of the protocol and determining what modifications should be made.

This decreased prioritization also may have been associated with the lack of a standing order to initiate SBTs. If a standing order had been placed, it may have resulted in higher protocol adherence and a decrease in time to the first SBT. This type of order was considered shortly after the start of the quality improvement project, but it was not implemented because it would have required a unit policy change, agreement among senior members of our division, 


\section{RT-Driven SBT Protocol IN THE PICU}

structural changes in our electronic health record, and a major change in clinical practice in our unit.

Another barrier we encountered during the course of this quality improvement project was that the critical care team may have unintentionally impaired the protocol. A few months after implementation, we observed that SBT orders were proactively being placed by the critical care team before RTs had an opportunity to screen. This may have occurred due to the critical care team's inability to adapt to this change in clinical practice or due to an attempt to simplify the process of initiating earlier SBTs to improve patient care. In any case, there was still no evidence of a statistically significant change in the initiation of earlier SBTs. Finally, because of the delicate nature of this protocol with regard to clinical practice, it lacked the formal educational process normally seen in quality improvement projects. This relaxed method, as well as the lack of the formal re-education during the project, may have contributed to RT non-adherence.

The low adherence rates highlighted opportunities for improvement. Any future modifications to the protocol must facilitate more flexibility and autonomy for the RT. For example, the critical care team should consider surrendering the assessment and ordering of SBTs. In future studies, we may consider allowing RTs to make the decision to start SBTs on the basis of predefined guidelines like those listed in our protocol, with the critical care team operating in a supportive role. This crucial change may facilitate future implementation of the protocol, as the RT may feel more autonomous and committed to performing and maintaining this protocol. If this protocol is instituted again with this change, we will plan to formalize the initial education and re-education of all clinicians involved in this protocol. We would focus the education on the goals for this project as well as communicate the measures we are tracking to identify if this protocol is an improvement. The re-education would primarily occur during high census months where adherence was previously noted to be the lowest. In addition, if the measures we are tracking show no improvement, despite an adequate adherence rate, we may reassess the content of the protocol and modify it to facilitate a higher adherence rate in a Plan-Do-StudyAct format. We believe that these changes would allow this protocol to be successful in our unit.

An RT-driven protocol may carry some risks. There was an increased risk of the use of noninvasive ventilation, which potentially could be considered an adverse effect. This could indicate a change of postextubation practices, but it may also indicate that patients who are recognized as ready for SBT initiation could be liberated too soon. ${ }^{17,18}$ Alternatively, one could argue that early extubation, even with the requirement of subsequent postextubation use of noninvasive ventilation, is preferable to a longer duration of invasive ventilation. This requires further study. In ad- dition, ventilator rate changes were physician-directed and not included in the protocol. Thus, the time for ventilator rate weaning may not have been performed in a sufficient amount of time and may have contributed to the deconditioning of the subject during disease recovery. This finding suggests that reliance on SBTs only for the purpose for extubation readiness may not take into account resolution of the patient's disease process and underlying deconditioning.

The main limitation of this study is that it this is a quality improvement project. Therefore it was not subject to randomization, making it difficult to assess the impact of the RT-driven protocol on initiation of SBTs. In addition, no surveys were administered during this project; thus, informal feedback was relied upon to ensure adherence, protocol comprehension, and any issues with the protocol itself. The protocol was limited by the method of SBT testing. Because previous studies varied in the way SBTs were performed, specifically with regard to pressure support adjustment to ETT size, we opted to create a hybrid of what was performed in previous studies but also to reflect our practices. ${ }^{9-12}$ There were no formal educational interventions applied, which likely affected adherence to the protocol. Finally, this project was limited in that only $56.4 \%$ of eligible patients were screened. There may have been bias on which patient the RT chose to screen, which could have impacted the outcome and balance measures. Future projects will focus on the barriers and eligibility criteria that can impair protocol adherence.

\section{Conclusions}

We successfully initiated an RT-driven protocol in an academic PICU in an environment where mechanical ventilator practice changes are difficult to achieve. It did not impact time of SBT initiation, potentially due to the difficulty in maintaining adherence over time. The success of future RT-driven pediatric weaning protocols in our institution will consist of maintaining staff awareness, increasing flexibility in respiratory therapy clinical decision making, and continuing to allow gradual changes in pediatric mechanical ventilation practices. This quality improvement project was the first step toward this goal and has laid the groundwork for future study.

\section{ACKNOWLEDGMENTS}

The authors thank Tammy M Angeletti, a clinical specialist from the Penn State Health Respiratory Care and Pulmonary Diagnostics department, for her contribution to the protocol.

\section{REFERENCES}

1. Blackwood B, Murray M, Chisakuta A, Cardwell CR, O'Halloran P. Protocolized versus non-protocolized weaning for reducing the du- 


\section{RT-Driven SBT PRotocol IN THE PICU}

ration of invasive mechanical ventilation in critically ill paediatric patients. Cochrane Database Syst Rev 2013;(7):CD009082.

2. Rivera R, Tibballs J. Complications of endotracheal intubation and mechanical ventilation in infants and children. Crit Care Med 1992; 20(2):193-199.

3. Grant MJ, Scoppettuolo LA, Wypij D, Curley MA; RESTORE Investigative Team. Prospective evaluation of sedation-related adverse events in pediatric patients ventilated for acute respiratory failure. Crit Care Med 2012;40(4):1317-1323.

4. Stambouly JJ, McLaughlin LL, Mandel FS, Boxer RA. Complications of care in a pediatric care unit: a prospective study. Intensive Care Med 1996;22(10):1098-1104.

5. Newth CJ, Venkatarman S, Wilson DF, Meert KL, Harrison R, Dean $\mathrm{JM}$, et al. Weaning and extubation readiness in pediatric patients. Pediatric Crit Care Med 2009;10(1):1-11.

6. Ely EW, Maker EM, Dunagan DP, Burke HL, Smith AC, Kelly PT, et al. Effect on the duration of mechanical ventilation of identifying patients capable of breathing spontaneously. N Engl J Med 1996; 335(25):1864-1869.

7. Robertson TE, Mann HJ, Hyzy R, Rogers A, Douglas I, Waxman $\mathrm{AB}$, Weinert $\mathrm{C}$, et al. Multicenter implementation of a consensusdeveloped, evidence-based spontaneous breathing trial protocol. Crit Care Med 2008;36(10):2753-2762.

8. Duke T, Kissoon N, Van Der Voort E. Pediatric Intensive Care: A Global Perspective. Rogers Textbook of Pediatric Intensive Care, 5th ed. Philadelphia, PA: Lippincott Wiliams and Wilkins. 2016;8.

9. Faustino, EV, Gedeit R, Schwarz AJ, Asaro LA, Wypij D, Curley MA; Randomized Evaluation of Sedation Titration for Respiratory Failure (RESTORE) Study Investigators: Accuracy of an extubation readiness test in predicting successful extubation in children with acute respiratory failure from lower respiratory tract disease. Crit Care Med 2017;45(1):94-102.
10. Foronda FK, Troster EJ, Farias JA, Barbas CS, Ferraro AA, Faria LS, et al. The impact of daily evaluation and spontaneous breathing test on the duration of pediatric mechanical ventilation: a randomized controlled trial. Crit Care Med 2011;39(11):2526-2533.

11. Randolph AG, Wypij D, Venkataraman ST, Hanson JH, Gedeit RG, Meert KL, et al. Effect of mechanical ventilator weaning protocols on respiratory outcomes in infants and children: a randomized controlled trial. JAMA 2002;288(20):2561-2568.

12. Schultz TR, Lin RJ, Watzman HM, Durning SM, Hales R, Woodson A, et al. Weaning children from mechanical ventilation: a prospective randomized trial of protocol-directed versus physician-directed weaning. Respir Care 2001;46(8):772-782.

13. Popernack ML, Thomas NJ, Lucking SE. Decreasing unplanned extubations: utilization of the Penn State Children's Hospital sedation algorithm. Pediatr Crit Care Med 2004;5(1):58-62.

14. Kollef MH, Shapiro SD, Silver P, St John RE, Prentice D, Sauer S, et al. A randomized, controlled trial of protocol-directed versus physician-directed weaning from mechanical ventilation. Crit Care Med 1997;25(4):567-574.

15. Farias JA, Alia I, Esteban A, Golubicki AN, Olazarri FA. Weaning from mechanical ventilation in pediatric intensive care patients. Intensive Care Med 1998;24(10):1070-1075.

16. Bock KR, Silver P, Rom M, Sagy M. Reduction in tracheal lumen due to endotracheal intubation and its calculated clinical significance. Chest 2000;118(2):468-472.

17. Gupta P, Kuperstock JE, Hashmi S, Arnolde V, Gossett JM, Prodhan $\mathrm{P}$, et al. Efficacy and predictors of success of noninvasive ventilation for prevention of extubation failure in critically ill children with heart disease. Pediatr Cardiol 2013;34(4):964-977.

18. Nava S, Gregoretti C, Fanfulla F, Squadrone E, Grassi M, Carlucci A, et al. Noninvasive ventilation to prevent respiratory failure after extubation in high-risk patients. Crit Care Med 2005;33(11): 2465-2470.

This article is approved for Continuing Respiratory Care Education credit. For information and to obtain your CRCE

(free to AARC members) visit

www.rcjournal.com

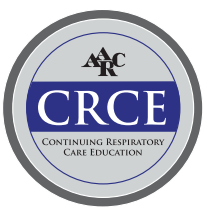

